\title{
The Mechanism of Calcitonin Gene-Related Peptide-Containing Nerve Innervation
}

\author{
Narumi Hashikawa-Hobara ${ }^{1, *,}$, Naoya Hashikawa ${ }^{1}$, Yoshito Zamami ${ }^{2}$, Shingo Takatori ${ }^{2}$, \\ and Hiromu Kawasaki ${ }^{2}$ \\ ${ }^{1}$ Department of Life Science, Okayama University of Science, 1-1 Ridai-cho, Okayama 700-0005, Japan \\ ${ }^{2}$ Department of Clinical Pharmaceutical Science, Graduate School of Medicine, Dentistry and Pharmaceutical Sciences, \\ Okayama University, 1-1-1 Tsushima-naka, Okayama 700-8530, Japan
}

Received February 28, 2012; Accepted April 2, 2012

\begin{abstract}
Calcitonin gene-related peptide (CGRP) is a major neurotransmitter and CGRPcontaining primary sensory neurons play an important role in nociception and potent vasodilation. CGRP-containing nerves in mesenteric arteries are decreased in pathological animal models (hypertension, diabetes, and atherosclerosis). In apolipoprotein E-knockout mice, which have atherosclerosis and peripheral sensory nerve defects, nerve growth factor (NGF)-mediated CGRP nerve facilitation was down-regulated, which may have been caused by the impairment of the Akt-NOcGMP pathway. In addition, NGF-mediated CGRP neurite outgrowth was decreased in fructoseinduced insulin-resistant rats. We recently discovered that renin-angiotensin inhibitors improved CGRP innervation in spontaneously hypertensive rats, indicating that rescuing CGRP nerve innervation might improve pathophysiological conditions. To find a novel reagent that facilitates CGRP nerves, a new model, phenol-injured perivascular nerve model rats, was established. Adrenomedullin, hepatocyte growth factor, and angiotensin II type 2 receptor activation induced CGRP nerve distribution in phenol-injured rats. Furthermore, in insulin-resistant model rats, the down-regulation of CGRP nerves was likely due to the depression of phosphoinositide 3-kinase (PI3K)-dependent Akt activation. Administration of candesartan improves CGRPergic function via the PI3K-Akt pathway in insulin-resistant rats. Thus, clarification of the mechanisms of CGRP nerve defects may constitute future therapeutic targets.
\end{abstract}

Keywords: calcitonin gene-related peptide-containing nerve, nerve growth factor-mediated neurite outgrowth, angiotensin II type 2 receptor, PI3K-Akt pathway

\section{Introduction}

Calcitonin gene-related peptide (CGRP) acts as a vasodilator neurotransmitter $(1-3)$. CGRP is distributed throughout the central and peripheral nervous systems and is located in areas involved in cardiovascular function (4). The dorsal root ganglia (DRG) are a prominent site of CGRP synthesis and contain the cell bodies of

*Corresponding author. hobara@dls.ous.ac.jp Published online in J-STAGE on May 17, 2012 (in advance) doi: $10.1254 /$ jphs.12R02CP primary afferent neurons that extend CGRP-containing (CGRPergic) nerves to the spinal cord and peripheral sites such as blood vessels (5). Perivascular nerves, along with endothelial cells, play an important role in the maintenance of vascular tone. The mesenteric artery, which comprises the largest vascular bed in the body, is innervated by many perivascular nerves such as sympathetic adrenergic nerves and non-adrenergic, non-cholinergic nerves, which include CGRPergic nerves (1). However, the distribution of CGRPergic neurons is still unclear in pathological animal models such as hypertension, atherosclerosis, and insulin-resistant diabetes.

${ }^{\S}$ This review article is written on the occasion of the author (Narumi Hashikawa-Hobara) receiving the 27th Award for Encouragement of Young Investigators from The Japanese Pharmacological Society in 2012 and on the basis of the targeted work. 


\section{Changes in distribution of CGRPergic in pathologi- cal animal models}

\subsection{Spontaneously hypertensive rats (SHR)}

The innervation and function of CGRPergic nerves in mesenteric resistance arteries of SHR decrease with age, which causes CGRPergic nerve remodeling (6). The distribution of CGRPergic nerves was decreased by agerelated changes in SHR. However, neuropeptide Y (NPY)-containing sympathetic adrenergic nerves were not affected by ageing. These results suggested that the remodeling of the CGRPergic innervation contributes to the development and maintenance of hypertension in SHR. Furthermore, long-term treatment with the angiotensin II (Ang II) type $1 \quad\left(\mathrm{AT}_{1}\right)$-receptor antagonist losartan prevented the CGRPergic nerve remodeling in SHR (6), implying that Ang II is an active substance that induces perivascular nerve remodeling via $\mathrm{AT}_{1}$ receptors. These studies led to the hypothesis that the restoration of CGRPergic nerve innervation after the blockade of $\mathrm{AT}_{1}$ receptors in SHR may be due to stimulation of Ang II type $2\left(\mathrm{AT}_{2}\right)$ receptors, which promote nerve regeneration.

\subsection{Apolipoprotein $\mathbf{E}$-deficient $\left(\operatorname{apo}^{-1}\right)$ mouse}

$\mathrm{ApoE}^{-/-}$mice spontaneously develop hypercholesterolemia and atherosclerotic lesions in large arteries and exhibit hypertension (7). ApoE ${ }^{-/-}$mice also have a reduced number of unmyelinated axons within the sciatic nerve and display reduced sensitivity to noxious thermal stimuli (8). Our previous study demonstrated that apoE $\mathrm{E}^{-/-}$ mice have a reduced sensitivity to noxious thermal stimuli in the hot plate test (9). Furthermore, the density of CGRPergic nerves was significantly reduced by approximately $50 \%$ compared to age-matched wild-type mice (9). To clarify the mechanisms of CGRPergic reduction, we used DRG neurons isolated from wild-type and apoE $^{-/-}$mice. We examined whether exogenous nerve growth factor (NGF) could affect the neuronal sprouting response of DRG cells. In the DRG of apoE $\mathrm{E}^{-/}$ mice, NGF-mediated CGRP-like immunoreactivity (LI)-positive neurite outgrowth was significantly lower compared to NGF-treated DRG cells in wild-type mice (9). However, the level of NGF-receptor mRNA in apo $\mathrm{E}^{-/-} \mathrm{DRG}$ cells was similar to that in wild-type mice (9). To clarify the mechanism by which NGF-mediated neurite outgrowth is impaired, we focused on the AktNO-cGMP pathway. Expression of phosphorylated Akt was significantly reduced in the DRG of apoE ${ }^{-/-}$mice (9). The NO donor, sodium nitroprusside (SNP) or $S$-nitroso- $N$-acetylpenicillamine, did not affect NGF-mediated neurite outgrowth in apoE $\mathrm{E}^{-/}$cultured DRG cells (9). However, 8-bromoguanosine 3',5'-cyclic monophosphate sodium salt n-hydrate (8-Br-cGMP), a cGMP analog, induced NGF-mediated neurite outgrowth facilitation similar to wild-type NGF-mediated neurite outgrowth levels (9). Furthermore, in apo $\mathrm{E}^{-/-} \mathrm{DRG}$, soluble guanylate cyclase (SGC) expression was significantly lower than in wild-type DRG (9). These results suggest that in apoE ${ }^{-/-}$mice, the Akt-NO-cGMP pathway is impaired and the impairment may be caused by NGF-mediated CGRP-LI-neurite outgrowth defects (Fig. 1).

\subsection{Fructose-induced insulin resistant rats}

Fructose drinking rats (FDR), a model of insulin resistance, develop hyperinsulinemia, but not hyperglycemia, which suppresses the function and innervation of CGRPergic nerves in the DRG $(10,11)$. The density of CGRPergic fibers in FDR was markedly decreased by approximately $20 \%$ compared to age-matched control rats. To investigate the mechanism of reduction of CGRPergic nerves in FDR, we focused on the NO-cGMP cascade. We examined whether exogenous NGF could affect the neuronal sprouting response of DRG cells. In the DRG of FDR, NGF-mediated CGRP-LI-positive neurite outgrowth was significantly lower when compared to the DRG of non-treated rats. To investigate whether exogenously induced $\mathrm{NO} / \mathrm{PKG}$ could affect the down-regulated NGF signaling pathway in DRG neurons of FDR, we examined effects of an NO donor and a PKG activator on nerve extension in the presence of NGF. The NO donor, SNP $(10 \mu \mathrm{M})$, did not affect NGF-induced neurite outgrowth. However, the activator of $\mathrm{PKG}, 8-\mathrm{Br}-$ cGMP, slightly reversed the reduction in neurite outgrowth caused by NGF to a similar amount as observed in control rats. These results suggested that the decrease in NGF-mediated CGRP-LI-positive neurite outgrowth was associated with the NO-cGMP signaling pathway in FDR (Fig. 1).

\section{Phenol-injured perivascular nerves model}

Based on the aforementioned findings, we proposed that the prevention and reversal of CGRPergic nerve remodeling might be therapeutic for hypertension or perivascular nerve disorder. Therefore, the present study was designed to establish an animal model for perivascular nerve remodeling with re-innervation and/or redistribution of adrenergic and CGRPergic nerves through the use of topical phenol treatment, which has been used to block peripheral nerve activity (12). Compared to shamoperated rats, treatment with phenol resulted in a $70 \%$ reduction in CGRP-LI nerves and a $40 \%$ reduction in NPY-LI nerves 7 days later, suggesting that phenol treatment causes non-selective damage to the perivascular nerves, both sympathetic adrenergic and CGRPergic 


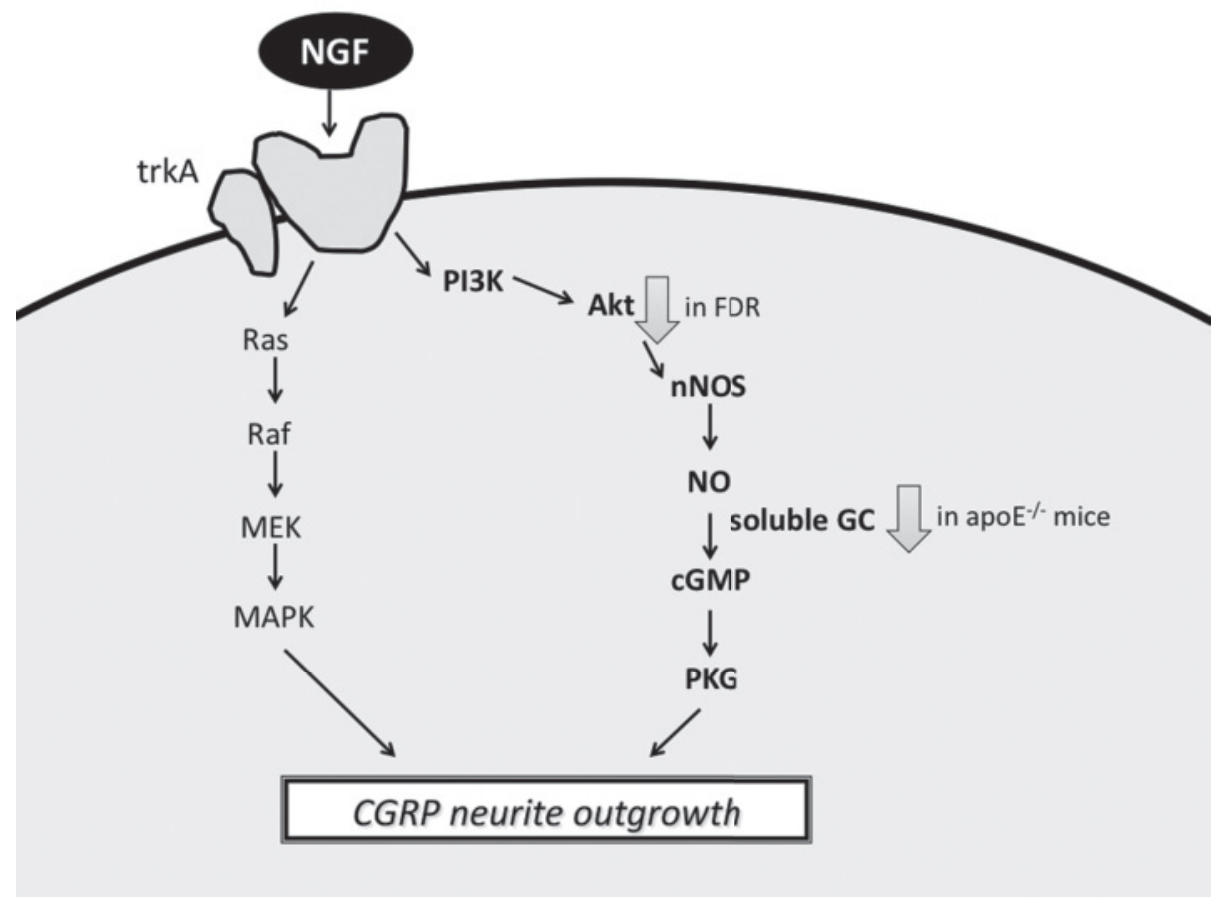

Fig. 1. Mechanism of CGRP-positive neurite outgrowth in DRG cells. NGF: nerve growth factor, trkA: tropomyosin-related kinase A, MAPK: mitogen-activated protein kinase, PI3K: phosphoinositide 3-kinase, FDR: fructose drinking rats, nNOS: neuronal nitric oxide synthase, NO: nitric oxide, GC: guanylate cyclase, cGMP: cyclic guanosine monophosphate, PKG: protein kinase G, CGRP: calcitonin gene-related peptide, apoE $\mathrm{E}^{-/}$: apolipoprotein $\mathrm{E}$ knockout.

ones (13). The density of CGRPergic nerves was almost abolished 3 days after phenol treatment, and this phenomenon lasted until day 14 , while the density of NPYLI nerves gradually decreased after the treatment (13). These findings suggest that CGRPergic nerves are more sensitive to phenol treatment. Furthermore, administration of NGF $(250 \mathrm{ng} / \mathrm{h})$, which was administered intraperitoneally for 7 days using an osmotic mini-pump immediately after topical phenol treatment, resulted in an increase in the density of both CGRP-LI and NPY-LI nerve fibers, which suggests that mesenteric perivascular nerves could regenerate and/or reinnervate tissues (13). Phenol-injured perivascular nerves model will provide a novel experimental paradigm for investigating the function and regeneration of perivascular nerves.

\section{Exploring novel substances that develop nerve reinnervation}

\subsection{Adrenomedullin}

Next, we searched for substances that promote nerve regeneration by using the phenol-injured perivascular nerves model. We focused on adrenomedulin, which has partial sequence homology with CGRP. We previously showed evidence of the colocalization of adrenomedullin and CGRP in the perivascular nerves and DRG (14).
Therefore, it is inferred that adrenomedullin, which is colocalized with CGRP in capsaicin-sensitive nerves, has neuronal activity such as nerve differentiation. Compared with saline treatment, adrenomedullin (360 or 1000 $\mathrm{ng} / \mathrm{h}$ ), which was administered intraperitoneally for 7 days using an osmotic mini-pump, significantly increased the density of CGRP-LI- and NPY-LI nerve fibers (15). These results suggested that adrenomedullin has a facilitating effect on the reinnervation of perivascular nerves as observed in NGF-treated nerves.

\subsection{Hepatocyte growth factor (HGF)}

HGF has been shown to have neurotrophic effects in the cerebral cortex, sensory neurons, and spinal cord motor neurons $(16-18)$. In the peripheral nervous system, HGF has also been reported to have a neurotrophic effect on adult motor neurons after axotomy of the hypoglossal nerves in vivo (19). However, it remains unclear whether HGF has trophic effects on peripheral perivascular nerves. HGF (30 $\mu \mathrm{g} / \mathrm{kg}$ per day) was administered for 7 days using an osmotic mini-pump after phenol treatment. HGF treatment significantly increased the density and number of CGRP-LI-containing nerve fibers compared to saline administration, while HGF did not affect the density of NPY-containing adrenergic nerve fibers (20). 


\section{3. $\mathrm{AT}_{2}$-receptor activation}

$\mathrm{AT}_{2}$ receptors are involved in neuronal differentiation of PC12W cells (21). Furthermore, there is a growing body of evidence that stimulation of $\mathrm{AT}_{2}$ receptors promotes cell differentiation and regeneration in neuronal tissues (22). $\mathrm{AT}_{2}$ receptors are drastically up-regulated under conditions such as tissue injury (23) or sciatic nerve axotomy (24). Several studies have suggested that Ang II, via the $\mathrm{AT}_{2}$ receptors, acts as a neurotrophic factor for peripheral neurons (25). Furthermore, long-term treatment with an $\mathrm{AT}_{1}$-receptor antagonist prevents CGRPergic nerve remodeling in SHR (6), implying that Ang II is an active substance that induces perivascular nerve remodeling via $\mathrm{AT}_{1}$ receptors. Thus, these studies led to the hypothesis that the restoration of CGRPergic nerve innervation after the blockade of $\mathrm{AT}_{1}$ receptors in SHR might be due to stimulation of $\mathrm{AT}_{2}$ receptors, which exert a neurotrophic effect on the reinnervation. Ang II (750 ng/kg per day) and PD123,319 (AT ${ }_{2}$-receptor antagonist, $10 \mathrm{mg} / \mathrm{kg}$ per day) were intraperitoneally administered for 7 days using osmotic mini-pumps immediately after topical phenol treatment. Losartan $\left(\mathrm{AT}_{1}\right.$-receptor antagonist) was administered in the drinking water $(0.025 \%)$. Co-administration of Ang II and losartan significantly increased the density of CGRP-LI fibers, but not NPY-LI fibers compared to the saline control (26). PD123,319 suppressed the increase in the density of CGRP-LI fibers by the co-administration of Ang II and losartan (26). These results suggest that selective stimulation of $\mathrm{AT}_{2}$ receptors by Ang II facilitates reinnervation of mesenteric perivascular CGRP-containing nerves.

\section{Effect of $\mathrm{AT}_{1}$-receptor blockade on CGRPergic disorder in fructose-induced insulin-resistant rats}

Because the activation of $\mathrm{AT}_{2}$ receptor facilitates reinnervation of mesenteric perivascular CGRPergic nerves in nerve-injured rats following phenol treatment (15), we hypothesized that in insulin-resistant rats, which have impaired peripheral CGRP nerve function $(10,11)$, neurite outgrowth may be affected via $\mathrm{AT}_{2}$-receptor activation. We examined whether the depression of CGRP nerves in $\mathrm{FDR}$ is related to $\mathrm{AT}_{2}$-receptor inactivation and whether treatment with the $\mathrm{AT}_{1}$-receptor antagonist candesartan cilexetil improves this depressed response. We found that the FDR developed insulin resistance and the density of CGRP-LI nerve fibers in the group of FDR was markedly decreased by approximately $20 \%$, compared with age-matched control rats (27). In contrast, administration of the $\mathrm{AT}_{1}$-receptor antagonist candesartan cilexetil increased the density of CGRP-LI nerve fibers to control levels (27). In addition, FDR developed the downregulation of both $\mathrm{AT}_{2}$-receptor neuronal function and phosphorylated Akt expression in DRG neurons (27). Candesartan improved neurite outgrowth in FDR, which was associated with the restoration of $\mathrm{AT}_{2}$ receptor and phosphorylated Akt expression (27). Akt is activated by insulin or various growth factors to function in a phosphoinositide 3-kinase (PI3K)-sensitive pathway. Furthermore, downregulation of $\mathrm{PI} 3 \mathrm{~K}$ inhibited $\mathrm{AT}_{2}$ receptor-mediated neurite outgrowth in control DRG cells, indicating that insulin resistance induces the downregulation of $\mathrm{AT}_{2}$-receptor function (27) because PI3K inhibition results in insulin resistance (28). In contrast, PI3K activation increased $\mathrm{AT}_{2}$ receptor-mediated neurite outgrowth and phosphorylated Akt expression in FDR DRG cells (27). These results suggest that the decrease in $\mathrm{AT}_{2}$ receptor-mediated neurite outgrowth in FDR is likely due to decreased PI3K-dependent Akt activation. Furthermore, candesartan improved $\mathrm{AT}_{2}$-receptor neuronal function and Akt phosphorylation, which were associated with insulin sensitivity in FDR (Fig. 2).

\section{Conclusion}

In pathological conditions, the function and innervation of CGRPergic nerves were decreased. NGF-mediated CGRP-positive neurite outgrowth, which was associated with NO-cGMP downregulation, was also reduced in pathological animal models such as apoE ${ }^{-/-}$mice or insulin-resistant rats. Interestingly, administration of adrenomedullin or $\mathrm{HGF}$ and also activation of $\mathrm{AT}_{2}$ receptor led to CGRP nerve elongation in phenol-injured rats. Furthermore, treatment with the $\mathrm{AT}_{1}$-receptor blocker candesartan improved CGRPergic function via the PI3K-Akt pathway in insulin-resistant rats. These results

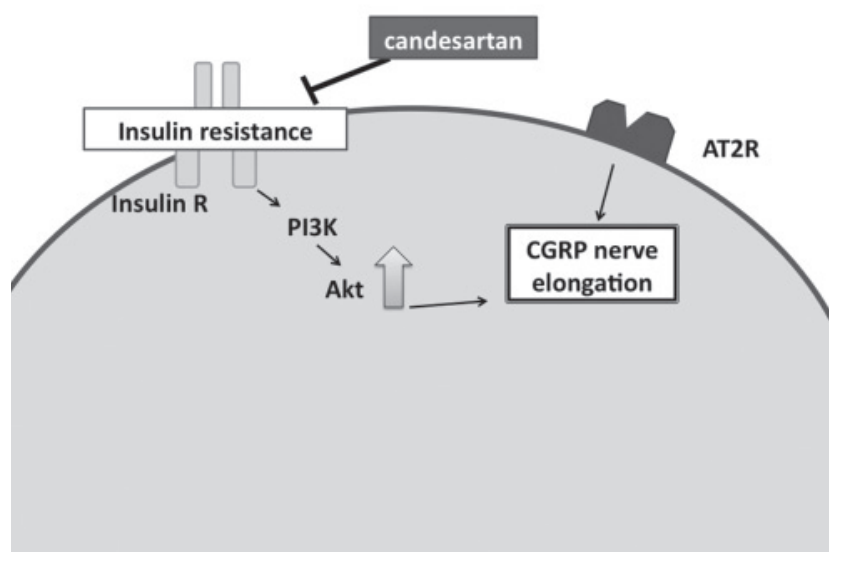

Fig. 2. Effect of candesartan treatment on CGRP-positive neurite outgrowth in DRG cells. AT2R: angiotensin II type 2 receptor, PI3K: phosphoinositide 3-kinase. 
suggest that clarifying the mechanisms of CGRP nerve down-regulation could contribute to the development of new therapeutic drugs for peripheral nerve injury.

\section{References}

1 Kawasaki H, Takasaki K, Saito A, Goto K. Calcitonin generelated peptide acts as a vasodilator neurotransmitter in mesenteric resistance vessels of the rat. Nature. 1988;335:164-167.

2 Kawasaki H, Nuki C, Saito A, Takasaki K. Role of calcitonin gene-related peptide containing nerves in the vascular adrenergic neurotransmission. J Pharmacol Exp Ther. 1990;252:403-409.

3 Kawasaki H, Nuki C, Saito A, Takasaki K. NPY modulates neurotransmission of CGRP-containing vasodilator nerves in rat mesenteric arteries. Am J Physiol. 1991;261:H683-H690.

4 Ishida-Yamamoto A, Tohyama M. Calcitonin gene-related peptide in the nervous system. Prog Neurobiol. 1989;33:335-386.

5 Marti E, Gibson SJ, Polak JM, Facer P, Springall DR, Van Aswegen $G$, et al. Ontogeny of peptide and amine-containing neurons in motor, sensory, and autonomic regions of rat and human spinal cord, dorsal root ganglia and rat skin. J Comp Neurol. 1987;266:332-359.

6 Hobara N, Gessei-Tsutsumi N, Goda M, Takayama F, Akiyama $\mathrm{S}$, Kurosaki Y, et al. Long-term inhibition of angiotensin prevents reduction of periarterial innervation of calcitonin gene-related peptide (CGRP)-containing nerves in spontaneously hypertensive rats. Hypertens Res. 2005;28:465-474.

7 Yang R, Powell-Braxton L, Ogaoawara AK, Dybdal N, Bunting $\mathrm{S}$, Ohneda $\mathrm{O}$, et al. Hypertension and endothelial dysfunction in apolipoprotein E knockout mice. Arterioscler Thromb Vasc Biol. 1999;19:2762-2768.

8 Fullerton SM, Strittmatter WJ, Matthew WD. Peripheral sensory nerve defects in apolipoprotein E knockout mice. Exp Neurol. 1998;153:156-163.

9 Hashikawa-Hobara N, Hashikawa N, Yutani C, Zamami Y, Jin X, Takatori S, et al. The Akt-nitric oxide-cGMP pathway contributes to nerve growth factor-mediated neurite outgrowth in apolipoprotein E knockout mice. J Pharmacol Exp Ther. 2011;338: 694-700.

10 Takatori S, Zamami Y, Yabumae N, Hanafusa N, Mio M, Egawa T, et al. Pioglitazone opposes neurogenic vascular dysfunction associated with chronic hyperinsulinaemia. $\mathrm{Br} \mathrm{J}$ Pharmacol. 2008;153:1388-1398.

11 Zamami Y, Takatori S, Hobara N, Yabumae N, Tangsucharit P, Jin $\mathrm{X}$, et al. Hyperinsulinemia induces hypertension associated with neurogenic vascular dysfunction resulting from abnormal perivascular innervations in rat mesenteric resistance arteries. Hypertens Res. 2011;34:1190-1196.

12 Wang Y, Bukoski RD. Use of acute phenolic denervation to show the neuronal dependence of $\mathrm{Ca}^{2+}$-induced relaxation of isolated arteries. Life Sci. 1999;64:887-894.

13 Hobara N, Goda M, Kitamura Y, Takayama F, Kawasaki H. Innervation and functional changes in mesenteric perivascular calcitonin gene-related peptide- and neuropeptide Y-containing nerves following topical phenol treatment. Neuroscience. 2006;141:1087-1099.

14 Hobara N, Nakamura A, Ohtsuka A, Narasaki M, Shibata K, Gomoita Y, et al. Distribution of adrenomedullin-containing perivascular nerves in the rat mesenteric artery. Peptides.
2004;25:589-599.

15 Hobara N, Goda M, Kitamura Y, Sendou T, Gomita Y, Kawasaki H. Adrenomedullin facilitates reinnervation of phenol-injured perivascular nerves in the rat mesenteric resistance artery. Neuroscience. 2007;144:721-730.

16 Honda S, Kagoshima M, Wanaka A, Tohyama M, Matsumoto K, Nakamura T. Localization and functional coupling of HGF and c-Met/HGF receptor in rat brain: implication as neurotrophic factor. Brain Res Mol Brain Res. 1995;32:197-210.

17 Ebens A, Brose K, Leonardo ED, Hanson MG Jr, Bladt F, Birchmeier C, et al. Hepatocyte growth factor/scatter factor is an axonal chemoattractant and a neurotrophic factor for spinal motor neurons. Neuron. 1996;17:1157-1172.

18 Hashimoto N, Yamanaka H, Fukuoka T, Dai Y, Obata K, Mashimo $\mathrm{T}$, et al. Expression of HGF and cMet in the peripheral nervous system of adult rats following sciatic nerve injury. Neuroreport. 2001;12:1403-1407.

19 Okura Y, Arimoto H, Tanuma N, Matsumoto K, Nakamura T, Yamashima T, et al. Analysis of neurotrophic effects of hepatocyte growth factor in the adult hypoglossal nerve axotomy model. Eur J Neurosci. 1999;11:4139-4144.

20 Hobara N, Yoshida N, Goda M, Yokomizo A, Kitamura Y, Sendou $\mathrm{T}$, et al. Neurotrophic effect of hepatic growth factor (HGF) on reinnervation of perivascular calcitonin gene-related peptide (CGRP)-containing nerves following phenol-induced nerve injury in the rat mesenteric artery. J Pharmacol Sci. 2008;108: 495-504.

21 Meffert S, Stoll M, Steckelings UM, Bottari SP, Unger T. The angiotensin AT2 receptor inhibits proliferation and promotes differentiation in PC12W cells. Mol Cell Endocrinol. 1996; 122:59-67.

22 Gasparo M, Catt KJ, Inagami T, Wright JW, Unger T. International union of pharmacology. XXIII. The angiotensin II receptors. Pharmacol Rev. 2000;52:415-472.

23 Viswanathan M, Saavedra JM. Expression of angiotensin AT2 receptors in the rat skin during wound healing. Peptides. 1992; 13:783-786.

24 Gallinat S, Yu M, Dorst A, Unger T, Herdegen T. Sciatic nerve transection evokes lasting up-regulation of angiotensin AT2 and AT1 receptor mRNA in adult rat dorsal root ganglia and sciatic nerves. Brain Res Mol Brain Res. 1998;57:111-122.

25 Lucius R, Gallinat S, Rosenstiel P, Herdegen T, Sievers J, Unger $\mathrm{T}$. The angiotensin II type 2 (AT2) receptor promotes axonal regeneration in the optic nerve of adult rats. J Exp Med. 1998; 188:661-670.

26 Hobara N, Goda M, Yoshida N, Takatori S, Kitamura Y, Mio M, et al. Angiotensin II type 2 receptors facilitate reinnervation of phenol-lesioned vascular calcitonin gene-related peptide-containing nerves in rat mesenteric arteries. Neuroscience. 2007; 150:730-741.

27 Hashikawa-Hobara N, Hashikawa N, Inoue Y, Sanda H, Zamami $\mathrm{Y}$, Takatori S, et al. Candesartan cilexetil improves angiotensin II type 2 receptor-mediated neurite outgrowth via the PI3K-Akt pathway in fructose-induced insulin resistant rats. Diabetes. 2012;61:925-932.

28 Zawalich WS, Zawalich KC. A link between insulin resistance and hyperinsulinemia: inhibitors of phosphatidylinositol 3-kinase augment glucose-induced insulin secretion from islets of lean, but not obese, rats. Endocrinology. 2000;141:3287-3295. 